\title{
Effect of duration on detection by the chromatic and achromatic systems
}

\author{
STEVEN H. SCHWARTZ \\ University of Alabama, Birmingham, Alabama \\ and \\ MICHAEL S. LOOP \\ University of Alabama, Birmingham, Alabama
}

\begin{abstract}
Detectability was determined as a function of stimulus duration for the chromatic and achromatic systems. For the achromatic system, detectability remains relatively constant for durations ranging from 43 to $1,000 \mathrm{msec}$, whereas for the chromatic system, detectability increases with duration up to about 500 msec. This suggests that the chromatic system has a more sustained output than the achromatic system.
\end{abstract}

Modern models of color vision suggest the existence of chromatic and achromatic systems, or channels, in the human visual system (Boynton, 1979). These channels have different temporal properties. The chromatic system displays relatively poor two-pulse resolution (Bowen, Lindsey, \& Smith, 1977), long critical durations (Regan \& Tyler, 1971), and high sensitivity to long-duration increments (King-Smith \& Carden, 1976). In contrast, the achromatic system shows comparatively high two-pulse resolution (Bowen et al., 1977), short critical durations (Regan \& Tyler, 1971), and high sensitivity to short-duration increments (King-Smith \& Carden, 1976). Moreover, temporal modulation transfer functions for the two systems are clearly different; the chromatic system shows a broad peak at relatively low temporal frequencies, whereas the achromatic system displays a relatively narrow peak at higher frequencies (Kelly \& van Norren, 1977). These differences lead one to suspect that the chromatic system responds to a step input in a relatively sustained fashion, whereas the achromatic system shows a transient response. Experimental evidence supporting this hypothesis has been presented by Tolhurst (1977), who demonstrated that a long-duration subthreshold pulse enhances sensitivity of the chromatic system for a longer period of time than it does for the achromatic system. Reaction time distributions (Schwartz \& Loop, 1982) obtained in response to

This research was supported by NIH Grants EY03303, EY03039, and RR05807 to the School of Optometry/The Medical Center of the University of Alabama in Birmingham. We thank Caroline Dunn for secretarial assistance and Ken Norris for assistance with the graphics. Please address correspondence to S. H. Schwartz, School of Optometry, University of California, Berkeley, CA 94720. long-duration near-threshold increments are also consisistent with this suggestion. In this paper, detectability is determined as a function of stimulus duration for both the chromatic and achromatic systems. This paradigm provides a straightforward demonstration of the different temporal properties of the two systems. The basic premise is that detection may occur at any time a system is active (i.e., responding). Since an increase in stimulus duration will prolong the activity of a sustained mechanism, detectability should be enhanced. To the extent that the chromatic system is sustained, increases in stimulus duration will result in increased detectability. However, for a transient mechanism, an increase in stimulus duration will not prolong the mechanism's output, and presumably will not increase detectability.

\section{METHOD}

\section{Subjects}

Two students (B.P., male; W.M., female) in their midtwenties, participated in the study. Both were experienced psychophysical observers who were naive with respect to the current study. The subjects demonstrated normal color vision on the Nagal anomaloscope (Pokorny, Smith, Verriest, \& Pinckers, 1979).

\section{Stimull}

The stimulus and background were presented in Maxwellian view, and both were $4 \mathrm{deg}$ in diameter. Both the stimulus and background originated from a single $12-\mathrm{V}$ tungsten-halogen bulb. Stimulus and background intensity were controlled by Iconel neutral density wedges. A 6-mm Uniblitz shutter was used to present stimuli with square-wave onset and of fset.

Stimuli predicted to reflect detection by the chromatic or achromatic system were obtained with conditions comparable to those utilized by King-Smith and Carden (1976). The background was white and had a retinal illuminance of 1000 Td (Nygaard \& Frumkes, 1982). The chromatic system was studied with increments of 440 or $620 \mathrm{~nm}$ (one-cavity interference filters), and white increments were used to study the achromatic system. 
Apparatus and Procedure

The subject depressed a telegraph key and solid state programming modules presented the stimulus following a random pretime period. The subject's task was to release the key in response to any change noted in the stimulus field. The subject had only one opportunity to respond to each stimulus, and the stimulus was not response terminated. In a single experimental session, which consisted of 100 or 150 trials, stimuli of 43-, 100-, 200-, 500-, and 1,000 -msec durations were presented in random sequence. The period following the stimuli was set so that the total duration of each trial was constant $(3,000 \mathrm{msec})$. In a given session, all stimuli were of the same chromaticity and luminance: only duration was varied. Data from three or four such sessions were combined to produce a plot of detectability as a function of duration.

\section{RESULTS AND CONCLUSIONS}

Figures 1 and 2 show functions relating detectability and duration for two subjects. Increments of 440 and $620 \mathrm{~nm}$ are predicted to reflect detection by the chromatic system. The functions obtained for these conditions show increasing detectability as the
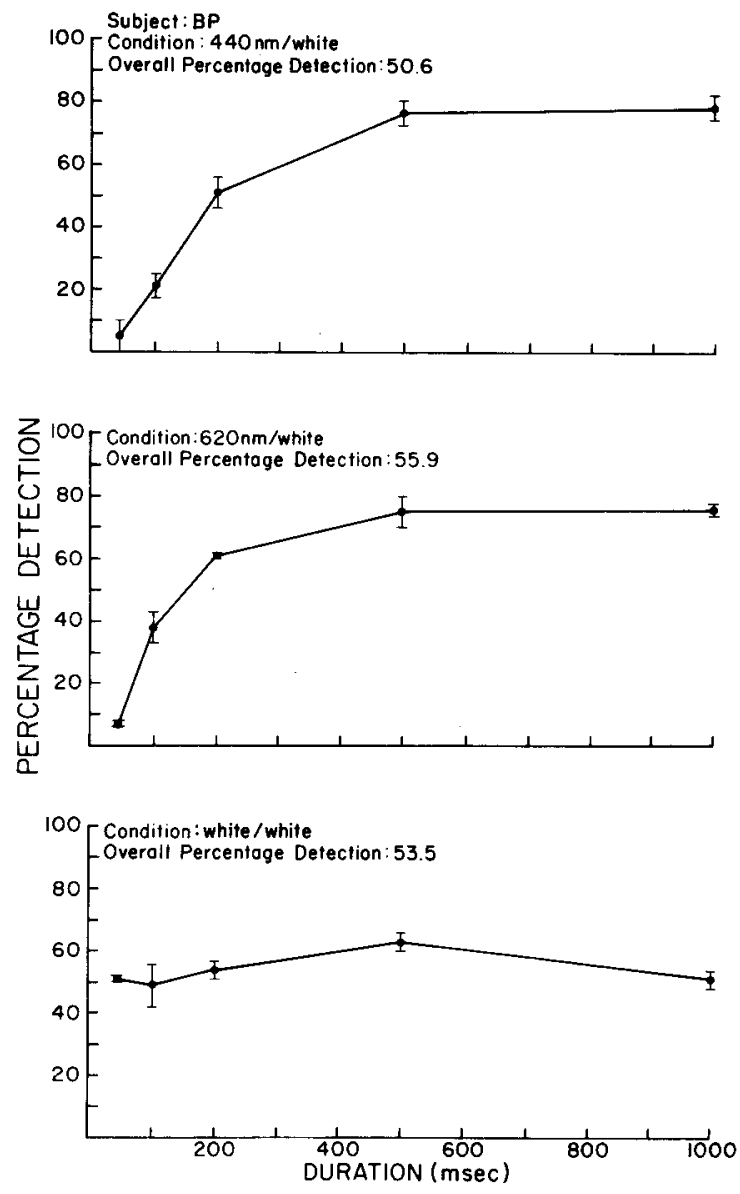

Figure 1. Percentage detection as a function of duration for Subject B.P. The top two graphs (increments of 440 and $620 \mathrm{~nm}$ ) were obtained with stimuli predicted to reflect detection by the chromatic system; the bottom graph (white increments) was obtained with stimuli predicted to reflect detection by the achromatic system. Each graph was obtained by combining the data obtained in four experimental sessions. Error bars show the SEMs.

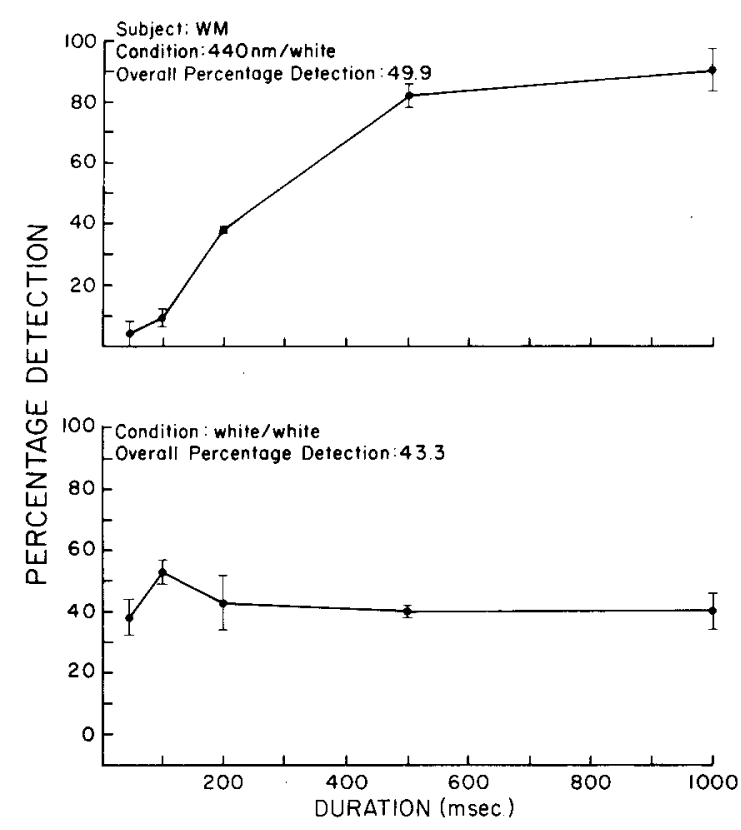

Figure 2. Percentage detection as a function of duration for subject W.M. See caption for Figure 1. The top graph represents data from three experimental sessions, and the bottom graph those from four sessions.

stimulus duration increases up to $500 \mathrm{msec}$; at $500 \mathrm{msec}$, detectability levels out. If we assume that detection may occur at any time the mechanism is active (i.e., responding), then the chromatic system is capable of an output of approximately $500 \mathrm{msec}$ in duration. As the stimulus duration is increased to $500 \mathrm{msec}$, detectability is enhanced, because these increases in duration cause the mechanism to respond for a longer period of time. Increasing the stimulus duration beyond $500 \mathrm{msec}$, however, does not increase detectability; the chormatic system, under these stimulus conditions, is presumably not capable of responding with an output greater than $500 \mathrm{msec}$ in duration.

Increments of white are predicted to reflect detection by the achromatic system. Figures 1 and 2 demonstrate, for both subjects, that increasing the stimulus duration from 43 to $1,000 \mathrm{msec}$ does not enhance stimulus detection. The achromatic channel responds with an output whose duration is less than that of the chromatic channel.

The primary assumption used in this analysis is that detection may occur at any time during a mechanism's response. Several models of visual detection that incorporate this suggestion have been proposed. For example, a stimulus may result in an increase in the mechanism's activity that is superimposed on a randomly fluctuating noise level (Tolhurst, 1975). An increase in the channel's response duration, with other properties of its response kept constant, should be expected to increase detectability. Also, a model 
that suggests that detection is dependent on the cumulative output of a basic mechanism (Lennie, 1981) predicts increasing detectability as a function of response duration.

The results obtained with this procedure are consistent with those of Tolhurst (1977) and Schwartz and Loop (1982), who suggested that the output of the chromatic system is sustained relative to that of the achromatic system. Schwartz and Loop determined reaction time (RT) distributions to longduration near-threshold increments. For the achromatic system, detection was tightly associated with stimulus onset and offset, whereas for the chromatic system, detection occurred over a longer period of time. However, with a $1,000-\mathrm{msec}$ stimulus, the chromatic system's RT distributions were not as long as the stimulus duration. Schwartz and Loop suggested that while the chromatic system's response is more sustained than that of the achromatic system, it is not fully sustained: the chromatic system's response, with long-duration stimuli, is less than $1,000 \mathrm{msec}$ in duration. This conclusion is supported by the results presented in this report. Curiously, the chromatic mechanism appears to have a less sustained output than the high-frequency channel described by Tolhurst (1975).

These differences in the temporal response properties of the chromatic and achromatic channels suggest that they represent distinct physiological pathways. Single-unit recordings in the primate retina (Gouras, 1968) and dorsal lateral geniculate nucleus (Schiller \& Malpeli, 1978) are consistent with this model. Neurons with a color-opponent organization tend to respond in a sustained fashion to a longduration step input. In contrast, noncolor opponent neurons display transient responses when presented with comparable stimuli.

\section{REFERENCES}

Bowen, R. W., Lindsey, D. T., \& SMith, V. C. (1977). Chromatic two-pulse resolution with and without luminance transients. Journal of the Optical Society of America, 67, 1501-1507.

Boynton, R. M. (1979). Human color vision. New York: Holt, Rinehart and Winston.

Gouras, P. (1968). Identification of cone mechanisms in monkey ganglion cells. Journal of Physiology (London), 199, 533-547.

Kelly, D. H., \& van Nornen, D. (1977). Two-band model of heterochromatic flicker. Journal of the Optical Society of America, 67, 1081-1091.

King-Smith, P. E., \& Carden, D. (1976). Luminance and opponent-color contributions to visual detection and adaptation and to temporal and spatial integration. Journal of the Optical Society of America, 66, 709-717.

LenNIE, P. (1981). The psychophysical basis of variations in visual latency. Vision Research, 21, 815-824.

Nygan d, R. W., \& Frumkes, T. E. (1982). Calibration of the retinal illuminance provided by Maxwellian views. Vision $R e-$ search, 22, 433-434.

Pokorny, J., Smith, V. C., Verriest, G., \& Pinckers, A. J. L. G. (1979). Congenital and acquired color vision defects. New York: Grune and Stratton.

REGAN, D., \& TYLer, C. W. (1971). Temporal summation and its limit for wavelength changes: An analog of Bloch's law for color vision. Journal of the Optical Society of America, 61, 1414-1421.

Schiller, P. H., \& Malpeli, J. G. (1978). Functional specificity of lateral geniculate nucleus laminae of the rhesus monkey. Journal of Neurophysiology, 41, 788-797.

Schwartz, S. H., \& LOOP, M. S. (1982). Evidence for transient luminance and quasi-sustained color mechanisms. Vision Research, 22, 445-447.

Tolmurst, D. J. (1975). Reaction times in the detection of gratings by human observers: A probabilistic mechanism. Vision Research, 15, 1143-1149.

Tolhurst, D. J. (1977). Colour-coding properties of sustained and transient channels in human vision. Nature, 266, 266-267.

(Manuscript received July 18, 1983; revision accepted for publication April 16, 1984.) 